\title{
Upgrade of liquid xenon calorimeter in MEG experiment with VUV sensitive MPPCs
}

\author{
Shinji OGAWA*广 \\ The University of Tokyo \\ E-mail: ogawa@icepp.s.u-tokyo.ac.jp
}

\begin{abstract}
The MEG II experiment is the upgrade of the MEG I experiment to search for the charged lepton flavor violating decay of muon, $\mu^{+} \rightarrow e^{+} \gamma$. The MEG II experiment is expected to reach a branching ratio sensitivity of $4 \times 10^{-14}$, which is one order of magnitude better than the sensitivity of the MEG I experiment. All detectors will be upgraded, aiming to significantly improve the resolutions to cope with twice or higher beam rate in MEG II. The performance of the liquid xenon (LXe) $\gamma$-ray detector will be greatly improved with a highly granular scintillation readout realized by replacing 216 photomultiplier tubes (PMT) on the $\gamma$-ray entrance face with 4092 multipixel photon counters (MPPC). For this purpose, we have developed a new type of MPPC which is sensitive to the LXe scintillation light in vaccum ultraviolet (VUV) range, in collaboration with Hamamatsu Photonics K. K. The performance of the VUV-sensitive MPPC will be presented, as well as the expected resolutions of the LXe detector in MEG II evaluated by Monte Carlo simulations based on the measured properties of the MPPCs.
\end{abstract}

Flavor Physics \& CP Violation 2015,

May 25-29, 2015

Nagoya, Japan

${ }^{*}$ Speaker.

${ }^{\dagger}$ on behalf of the MEG II collaboration 


\section{Introduction}

Lepton flavor violating decay of muon, $\mu^{+} \rightarrow e^{+} \gamma$, is prohibited in the standard model. Neutrino oscillation can cause lepton flavor violation, but its branching ratio is too small $\left(\sim O\left(10^{-54}\right)\right)$ to detect. In contrast, some of the models beyond standard model such as SUSY-GUT and SUSY seesaw predict a detectable branching ratio $\left(\sim O\left(10^{-14}\right)\right)$ of $\mu^{+} \rightarrow e^{+} \gamma$. The upper limit of the branching ratio of this decay at $90 \%$ confidence level is $5.7 \times 10^{-13}$ given by MEG experiment [1] . MEG II experiment is the upgrade of the MEG I experiment. Target sensitivity is $4 \times 10^{-14}$, which is one order of magnitude better than the sensitivity of MEG I. Accidental background of the high energy positron and $\gamma$-ray is the dominant background, so all detectors will be upgraded to cope with the higher background rate caused by the twice or higher beam rate in MEG II. Resolutions of all detectors are designed to be improved by a factor of 2 . In addition to that, efficiency of the positron will also be improved by a factor of 2 .

\section{Upgrade of the LXe detector}

In the MEG I experiment, a liquid xenon (LXe) detector was used to measure the position, energy, and timing of $52.8 \mathrm{MeV} \gamma$-ray coming from the signal event. To detect the scintillation light in vaccum ultraviolet (VUV) range from $\gamma$-ray, 846 2-inch photomultiplier tubes (PMT) were placed around the liquid xenon. Conversion points of many of the $\gamma$-ray were located around the $\gamma$ entrance face of the detector. For these shallow events, photon collection efficiency highly depended on the relative position of the conversion point and PMT, and this limited the position and energy resolution in MEG I. In the MEG II experiment, 216 PMTs which were located in the $\gamma$ entrance face will be replaced with 4092 multi-pixel photon counters (MPPC) (Fig.1). The position and energy resolution will be improved thanks to the highly granular scintillation readout and more uniform photon collection, realized by this replacement. The detection efficiency to the $\gamma$-ray from signal event will also be improved as the amount of material of the entrance face will be reduced. We also plan to change the layout of PMTs on the lateral face. The $\gamma$ entrance face is extended by $20 \%$ along the beam axis to reduce the energy leakage for the events near the lateral face. PMTs on the lateral face will be placed as their photo-cathodes become parallel to the lateral face to reduce the effect of the shower fluctuation.

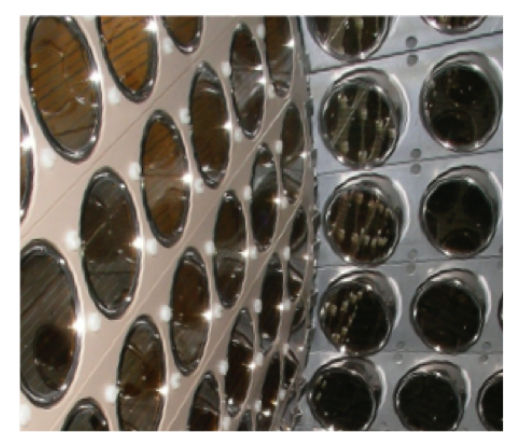

Figure 1: Left: LXe detector in MEG I, Right: LXe detector in MEG II (CG)
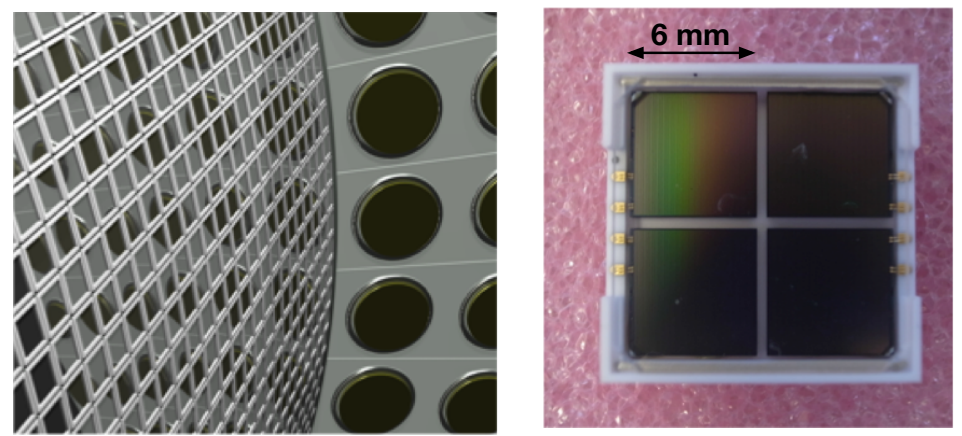

Figure 2: VUV-sensitive large area MPPC 
There are several requirements for MPPC used in the MEG II LXe detector. First, it can be operated in LXe. Second, it must be sensitive to scintillation light in VUV range. Third, it must have large area to keep the number of readout channels manageable. We have developed MPPC which meets these requirements in collaboration with Hamamatsu Photonics K. K.. Protection coating of MPPC is removed because VUV light have a short attenuation length in silicon. Crosstalk and afterpulse suppression technique is implemented which enables us to use it in higher voltage and to gain higher photon detection efficiency (PDE). Signal waveform with long time constant caused by the large capacitance from the large sensitive area could be a problem both for timing resolution and pileup elimination performance. To avoid this, we placed $46 \times 6 \mathrm{~mm}^{2}$ MPPC chips to one package (Fig.2), and their signals are read as one channel, by connecting 4 MPPC chips in series.

Signal transmission methods for more than 4000 channels have been developed. MPPCs are mounted on Printed Circuit Boards (PCB), which are not only for the signal transmission but also for the series connection and alignment. PCB based feedthrough have also been developed to realize the high density signal transmission through the cryostat wall. For both PCBs, "co-axial like" layer structure is implemented to shield signal line from outside, to have $50 \Omega$ impedance, and to minimize the crosstalk effect from the adjacent channels.

\section{Performance test of MPPC}

We performed a detailed test for small number of MPPCs in LXe. The setup of the measurement is shown in Fig.3. LED light and scintillation light from an $\alpha$ source were used as a light source. We can observe a clear 1 photoelectron (1 p.e.) peak in the charge distribution (Fig.4) thanks to the good gain uniformity between cells and low dark signal rate at LXe temperature. Gain is measured to be around $8 \times 10^{5}$ at $7 \mathrm{~V}$ over voltage with series connection. Crosstalk and afterpulse probability are calculated from charge distribution, by assuming Poisson distribution for the LED light, and measured to be only $25 \%$ at $7 \mathrm{~V}$ over voltage thanks to the suppression technique. Short waveform time constant is confirmed to the 1 p.e. signal thanks to the series connection.

We measured PDE to the scintillation light from the observed number of photoelectrons from the $\alpha$ source. Expected number of photons arrived at MPPC are calculated from energy of $\alpha$ particle and solid angle. Effect of the crosstalk and afterpulse is corrected. Measured PDE at $7 \mathrm{~V}$ over voltage is from $16 \%$ to $27 \%$ (Fig.5), and large systematics is thought to be coming from geometries of the setup. Energy resolution for the scintillation light is also measured and resolution down to $1 \%$ level, which is near the goal of our final detector, is confirmed.
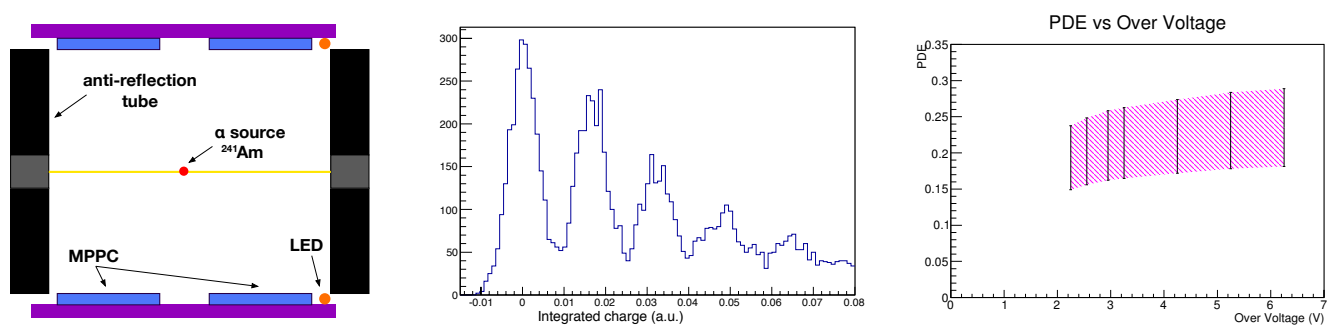

Figure 3: Setup for the small Figure 4: Charge distribution for Figure 5: PDE as a function of over sample test in LXe

LED light with series connection voltage 
In addition to the small sample test, we did the mass test for 600 MPPCs at room temperature, and no dead chip were found in this test. Mass test for 600 MPPCs have also been done in LXe. Signal readout was done by using prototypes of PCB, feedthrough, and cable. We could see the signal from MPPCs, except for 5\% of them. Most of these bad channels were found to be caused by bad connections in the cables and PCBs. Assembly procedure and design of the cable connector and PCB have been modified in order not to have the same problem in the final detector.

\section{Expected performance of the LXe detector}

Performance of the final detector has been estimated by using the Monte Carlo simulation based on the measured properties of MPPCs such as gain, PDE, waveform of 1 p.e., crosstalk probability, and afterpulse probability. Charge and timing for each MPPC and PMT are calculated from a simulated waveform, and used to reconstruct the position, energy, and timing of the $\gamma$-ray. Reconstruction algorithm is based on that in MEG I, and optimized to exploit the advantages of MPPC.

Detection efficiency for the $\gamma$-ray from the signal event is improved from $63 \%$ to $69 \%$. Improvement of the position resolution is expected especially for the shallow event thanks to the high granularity readout of the scintillation light. Energy resolution is also expected to improve thanks to the better uniformity of the photon collection efficiency. Timing resolution is estimated to be $60 \mathrm{ps}$, and small improvement is expected.

\section{Conclusion}

MEG II experiment is the upgrade of MEG I and searches for $\mu^{+} \rightarrow e^{+} \gamma$ with the branching ratio sensitivity of $4 \times 10^{-14}$. LXe detector will be upgraded, by replacing 216 PMTs on the $\gamma$ entrance face with 4092 MPPCs. For this purpose, VUV-sensitive MPPC has been developed and good performance is confirmed in LXe. By using the Monte Carlo simulation based on the measured properties of MPPC, position and energy resolution are estimated to be improved by a factor of 2 .

\section{References}

[1] J.Adam et al., New constraint on the existence of the $\mu^{+} \rightarrow e^{+} \gamma$ decay, Phys. Rev. Lett. 110,201801.
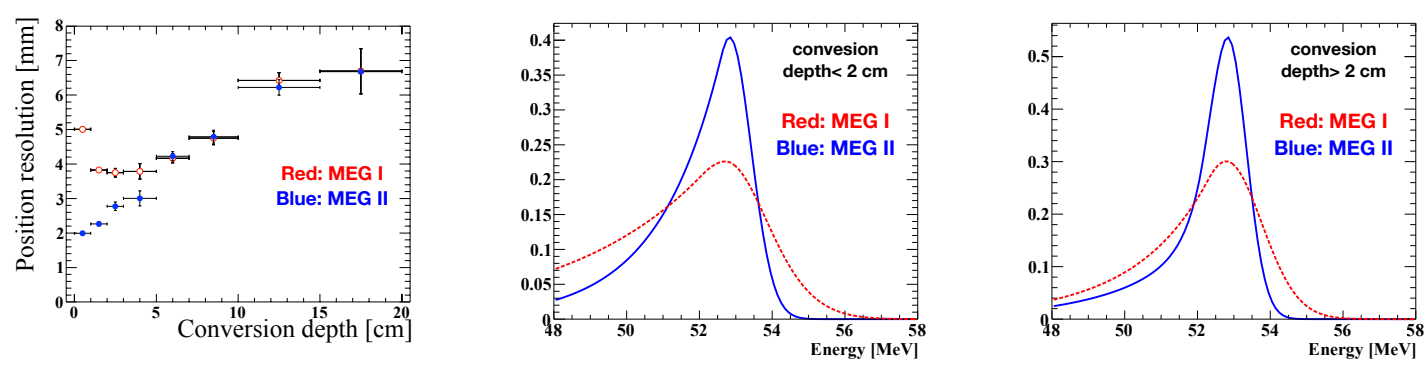

Figure 6: Position resolution as a Figure 7: Reconstructed energy distribution for signal $\gamma$-ray for converfunction of conversion depth sion depth $<2 \mathrm{~cm}$ (left) and for depth $>2 \mathrm{~cm}$ (right) 